PAPER

\title{
Propagation of fast and slow waves in cancellous bone: Comparative study of simulation and experiment
}

\author{
Yoshiki Nagatani ${ }^{1, *}$, Katsunori Mizuno ${ }^{2}$, Takashi Saeki ${ }^{2}$, \\ Mami Matsukawa ${ }^{3, \dagger}$, Takefumi Sakaguchi ${ }^{4}$ and Hiroshi Hosoi ${ }^{4}$ \\ ${ }^{1}$ Department of Electronic Engineering, Kobe City College of Technology \\ ${ }^{2}$ Graduate School of Engineering, Doshisha University \\ ${ }^{3}$ Faculty of Life and Medical Sciences, Graduate School of Engineering, Doshisha University \\ ${ }^{4}$ Department of Otorhinolaryngology-Head and Neck Surgery, Faculty of Medicine, \\ Nara Medical University
}

(Received 31 July 2008, Accepted for publication 23 January 2009)

\begin{abstract}
Comparative study of longitudinal wave propagation in cancellous bone was performed. We simulated wave propagation with the finite-difference time-domain (FDTD) method using a threedimensional X-ray computer tomography (CT) model of an actual cancellous bone. We also experimentally measured the waves that propagated in an identical specimen under similar conditions. The speeds of fast waves and the amplitudes of fast and slow waves at small ROIs (region of interest) in the specimen were examined. We found good correlations in fast wave speed and wave amplitudes between simulated and measured data. The peak amplitude ratio of simulated two waves was also similar with the experimental results. These results show the importance of FDTD simulation to understand the wave propagation phenomena in the complicated medium.
\end{abstract}

Keywords: Cancellous bone, Fast wave, Slow wave, X-ray CT, Three-dimensional elastic FDTD method

PACS number: 43.35.+d, 43.80.+p [doi:10.1250/ast.30.257]

\section{INTRODUCTION}

Considering the aging of Japanese society, demand is growing for a more reliable and less onerous method of diagnosing osteoporosis. Ultrasonic diagnosis systems are considered a powerful tool, because ultrasonic waves strongly reflect the elasticity. The current in vivo ultrasonic diagnosis technique is the "Speed Of Sound/Broadband Ultrasound Attenuation" (SOS/BUA) method [1]; however, as a simple measurement of averaged ultrasonic wave speed and attenuation of whole bone in the wave propagation path, this method does not really reflect the precise bone structure and the amount of minerals present.

In order to improve the accuracy of ultrasonic diagnosis system, our group has proposed a new approach to evaluate bone that utilizes the separation of longitudinal waves as they pass through cancellous bone [2-4]. We have reported the separation of longitudinal waves into fast and slow waves, a phenomenon that is especially dependent upon the

\footnotetext{
*e-mail: nagatani@kobe-kosen.ac.jp

†e-mail: mmatsuka@mail.doshisha.ac.jp
}

alignment of bone trabecula in cancellous bone [5-7]. It is hoped that this interesting phenomenon will become a powerful tool in the diagnosis of osteoporosis because the wave propagation behavior apparently depends on the bone structure. However, the details of wave propagation in cancellous bone have not yet been examined, because of the structural complexity and inhomogeneity.

Several theoretical approaches have been taken to elucidate the phenomenon of wave propagation in porous material. In Biot's theory [8], or the multi-layer model [9], the porous structure is described according to several parameters in an effort to predict the generation of fast and slow waves in cancellous bone. Hosokawa has reported the separation of waves by means of a numerical solution using Biot's theory with the finite-difference method [10]; however, difficulties in bone analysis remain because of the limited applicability of Biot's theory to cancellous bone $[11,12]$, which exhibits significant anisotropy and inhomogeneity.

The difficulties of analytic evaluation have led to the direct approach of attempting to solve the wave equation. If an exact simulation of wave propagation were to succeed, 
it would give us a visual image of complicated wave propagation in the bone. This would be helpful for constructing a novel and optimum in vivo ultrasonic measurement system [13].

Using a three-dimensional (3-D) synchrotron microtomograph of actual trabecula, Bossy et al. [14] and Padilla et al. [15] reported the generation of fast and slow waves using finite-difference time-domain (FDTD) simulation. Haïat et al. [16] investigated the influence of trabecular bone microstructure and material properties on SOS/BUA parameters using numerical simulations. However, a quantitative comparison between simulation and experimental results has not been investigated. Therefore, our group sought to confirm the applicability of the elastic FDTD method to the simulation of wave propagation in cancellous bone using three-dimensional X-ray CT images $[17,18]$ by focusing on the comparative studies between the FDTD simulation and experimental data. The tendency of the simulated results showed good agreement with experimental data; however, there were still a problem due to the lack of spatial resolution of simulation model [17]. Recent investigations of Padilla et al. [19] and Jenson et al. [20] show the similar tendency of SOS (or phase velocity) and BUA between simulated and experimental results; however, they have not examined the characteristics of twowave phenomenon.

In this study, therefore, we simulated wave propagation in a cancellous bone with the elastic FDTD method using a more precise three-dimensional X-ray CT model of an actual cancellous bone. We also experimentally measured the waves that propagated in an identical specimen under similar conditions in order to investigate the adequacy of the simulation technique, especially focusing on the twowave phenomenon.

\section{MEASUREMENT}

\subsection{Experimental Setup}

For this comparative study, the waves propagated through the bone were experimentally measured with PVDF transducers. The size of the bovine cancellous bone used was $20 \times 20 \times 9 \mathrm{~mm}^{3}$. Figure 1 shows the photograph of the bone specimen. The specimen was obtained from the head of 36-month-old bovine femur.

During the measurements, the bone specimen was immersed in degassed water at room temperature. Before measurement, the air in the porous structure was eliminated by degassing. The geometric focal length of the star-shaped concave PVDF transmitter (custom-made by TORAY, Tokyo, Japan) was $40 \mathrm{~mm}$ and the radius was $10 \mathrm{~mm}$. The surface of the transmitter was star-shaped in order to decrease the edge effect [21]. In water, the beam width at half maximum value of the wave amplitude was approximately $1.5 \mathrm{~mm}$ at the focal point [21]. The transmitted

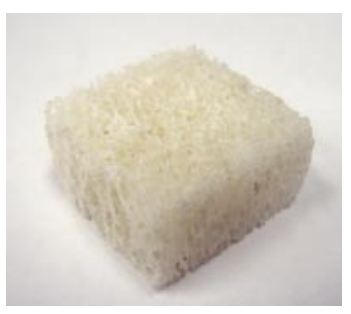

Fig. 1 Photograph of the specimen. The bone marrow inside the cancellous bone was removed.

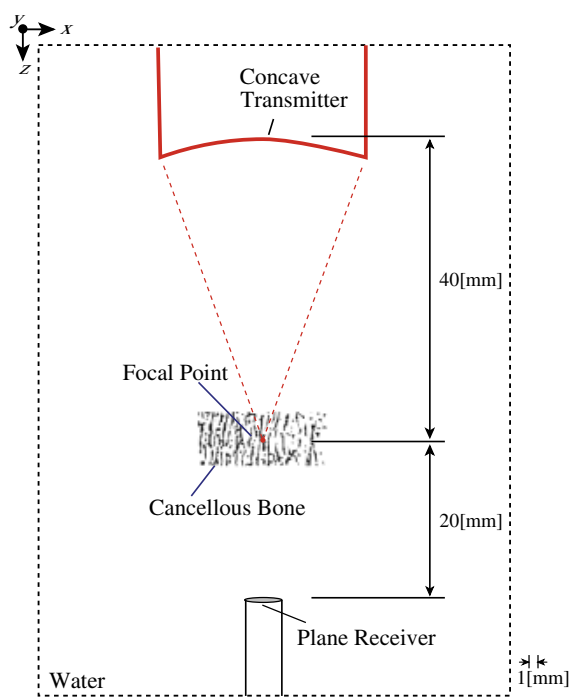

Fig. 2 Schematic representation near the specimen of the measurement system. The inside porous area and outside the specimen was filled with degassed water.

wave focused in the specimen at the mid point of the thickness. The radius of the plane PVDF receiver (homemade with PVDF film) was $2.5 \mathrm{~mm}$. Figure 2 shows the measurement system. In this measurement, the bone specimen was fully immersed in water. The $z$-axis position of the transmitter was fixed to maintain the focal point at the mid point of the specimen thickness without consideration of refraction. The distribution of the wave properties was investigated by changing the positions of the transmitter and receiver along the $x-y$ plane. They were moved in 1-millimeter-increments. The total number of measured points was 144 .

A single sinusoidal wave at $1 \mathrm{MHz}, 5 \mathrm{Vp}$-p from a function generator (WF1945, NF Corporation, Kanagawa, Japan) was amplified by a $20-\mathrm{dB}$ power amplifier (4055, NF Corporation, Kanagawa, Japan), and applied to the transmitter. By changing the positions of the specimen along the $x-y$ plane, we investigated the distribution of wave properties with a digital oscilloscope (TDS 524A, Tektronix Inc., Oregon, United States) with 40-dB preamplifier (5307, NF Corporation, Kanagawa, Japan). 

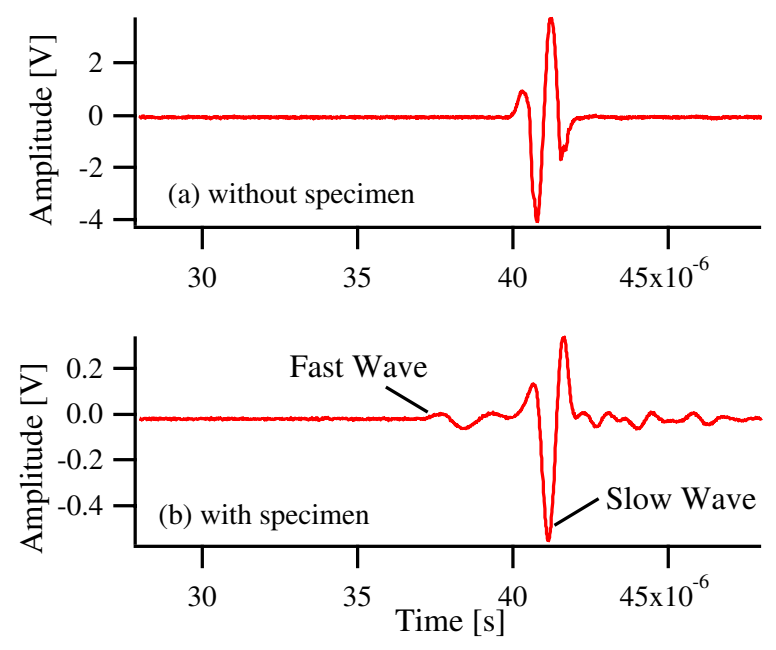

Fig. 3 Experimentally observed waveforms. Waves pass through the experimental setup, (a) without the specimen, or (b) with the specimen.

\subsection{Experimental Results}

Examples of the experimentally observed waveforms are shown in Figs. 3(a) and (b). Figure 3(a) shows the result when the transmitted wave passes only through water; Figure 3(b) shows the result when the transmitted wave is focused on the center portion of the specimen. In Fig. 3(b), the separation into fast and slow waves can be seen. Here, we adopted the first positive peak as the fast wave amplitude and the maximum amplitude of whole waveform as the slow wave amplitude.

\section{SIMULATION}

\subsection{Elastic FDTD Method}

The followings are the governing equations for the 3dimensional elastic FDTD method [22,23] for the isotropic medium. Deputized equations related to the $x$ direction are:

$$
\begin{gathered}
\frac{\partial \sigma_{x x}}{\partial t}=(\lambda+2 \mu) \frac{\partial v_{x}}{\partial x}+\lambda \frac{\partial v_{y}}{\partial y}+\lambda \frac{\partial v_{z}}{\partial z}, \\
\frac{\partial \sigma_{x y}}{\partial t}=\mu\left(\frac{\partial v_{x}}{\partial y}+\frac{\partial v_{y}}{\partial x}\right), \\
\frac{\partial v_{x}}{\partial t}=\frac{1}{\rho}\left(\frac{\partial \sigma_{x x}}{\partial x}+\frac{\partial \sigma_{x y}}{\partial y}+\frac{\partial \sigma_{z x}}{\partial z}\right),
\end{gathered}
$$

where $\sigma_{x x}$ and $\sigma_{x y}$ are normal and shear stresses, $v_{x}$ is particle velocity, $\lambda$ and $\mu$ are Lame's coefficients, and $\rho$ is the density of the medium. These equations are digitized using the central difference method. Figure 4 shows the configuration of the parameters of FDTD simulation. The stress and particle velocity were calculated alternately both in the spatial and time domains, which is called "the leapfrog method." Absorption layers were implemented on each end face of the model [24]. Making use of the advantage of simulation, we set the attenuation of the values of the normal and shear stresses in each calculation

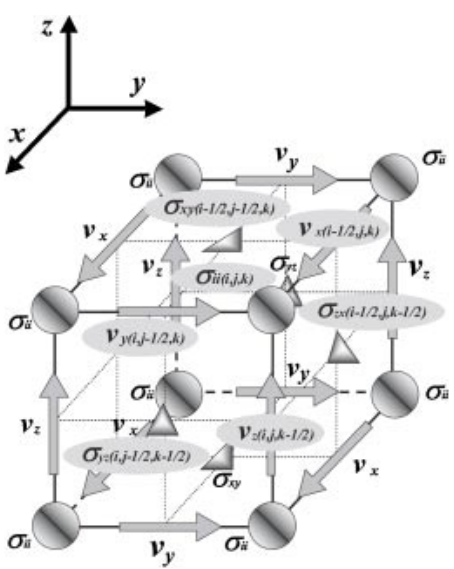

Fig. 4 The spatial configuration of the parameters of FDTD simulation.
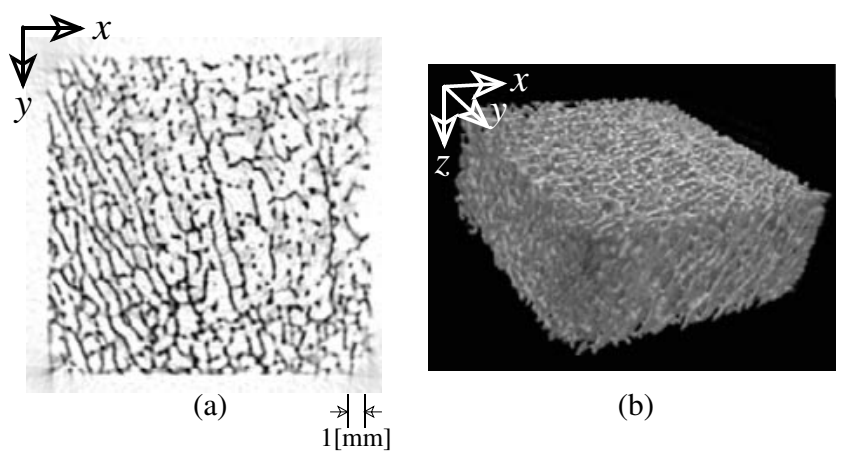

(b)

Fig. 5 A specimen of bovine cancellous bone used in the simulation and experiment. (a) One slice of the 2-D $\mathrm{X}$-ray CT image before binarization. (b) 3-D reconstructed model of cancellous bone after binarization.

step of wave propagation, considering $d x$ (spatial resolution). The attenuation for each step was determined from the experimentally observed longitudinal attenuation value of cortical bone at $1 \mathrm{MHz}[25,26]$. The attenuation used did not exhibit frequency dispersion. In this simulation, the elastic anisotropy of the solid portion was not considered.

\subsection{Simulation Model}

The wave propagation was simulated using the identical bone specimen that was experimentally measured in chapter 2. Figures 5(a) and (b) are X-ray micro-focus CT images (MCT-12505MF, Hitachi Medical, Tokyo, Japan). The spatial resolution of the CT images was $64.5 \mu \mathrm{m}$. This field was smaller than the experimental measurement area due to the memory limitaion of computer. The values of each point in the CT images were binarized in order to allow separation of the ambiguous border between the solid portions (trabecula) and liquid portions at a specific threshold. The threshold was determined as the median value of the grayscale tones of the image. 


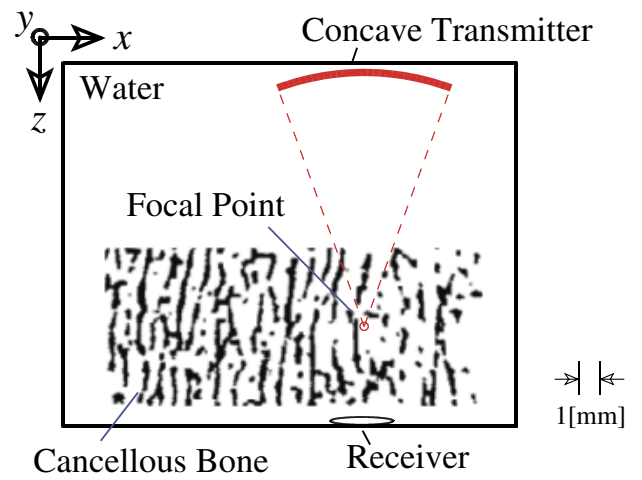

Fig. 6 The FDTD simulation model. The concave transmitter faces the specimen immersed in water. Absorption layers were implemented on each end face of the model.

The total simulation field was $23 \times 23 \times 18 \mathrm{~mm}^{3}$ with cube lattice of $64.5 \mu \mathrm{m}$. The time increment of simulation was $5 \mathrm{~ns}$. As the initial particle velocity in $z$ direction at the surface of the concave source shown in Fig. 6, the single sinusoidal wave at $1 \mathrm{MHz}$ was used. The Hanning window whose length was $1 \mu \mathrm{s}$ was applied to prevent the discontinuity of the waveform.

In this simulation, the surface of the transmitter was star-shaped in order to decrease the edge effect [21]. This comes from the actual transducer used in the experiments. The surface polygon had 16 apexes. The outer radius of the star shape was $2.5 \mathrm{~mm}$ and the inside radius was $1.25 \mathrm{~mm}$. The geometric focal length of concave transmitter was $10 \mathrm{~mm}$. Because of the lack of memory capacity of the computer, the configuration of the transmitter in this simulation was set proportional to the actual experimental system. The receiver was $1.5 \mathrm{~mm}$ in radius, and the results of the simulated sound pressures were obtained from the integrated values on the receiver surface, where the sound waves were not reflected nor interrupted. The transmitter and the receiver have the acoustical axis in common.

In this model, the bone specimen was fully immersed in water. The $z$-axis position of the transmitter was fixed to maintain the focal point at the mid point of the specimen thickness without considering the refraction. The distribution of the wave propagation was investigated by changing the positions of the transmitter and receiver in the $x-y$ plane. They were moved in about 1-millimeter-increments. The total number of simulated points was 144 .

The experimentally observed values in the bovine cortical bone were used as the wave speed and attenuation in the trabeculae part of the cancellous bone. Yamato et al. [27-29] reported that the speed of the longitudinal wave in cortical bone varied from 4,000 to $4,400 \mathrm{~m} / \mathrm{s}$. In this study, the simulations were performed at the longitudinal wave speed of $4,400 \mathrm{~m} / \mathrm{s}$ because much higher velocities were reported in the trabeculae in the $\mathrm{GHz}$ range [30]. In the
Table 1 Parameters used in the FDTD simulation.

\begin{tabular}{cccc}
\hline \multicolumn{2}{c}{ Material } & Water & Bone trabecula \\
\hline Density $\left[10^{3} \mathrm{~kg} / \mathrm{m}^{3}\right]$ & 1.0 & 2.0 \\
\hline Velocity $[\mathrm{m} / \mathrm{s}]$ & Longitudinal & 1,500 & 4,400 \\
& Shear & - & 2,200 \\
\hline Lame's constant [GPa] & $\lambda$ & 2.2 & 20.0 \\
& $\mu$ & - & 9.4 \\
\hline Attenuation [neper $/ \mathrm{m}]$ & Longitudinal & 0.025 & 80 \\
& Shear & - & 160 \\
\hline
\end{tabular}

simulation, the speed of shear wave was the estimated value, assuming Poisson's ratio of 0.34 [2]. We adopted the experimentally observed attenuation in the cortical bone as the attenuation coefficients of longitudinal wave in trabeculae $[25,26]$. The attenuation coefficient of the shear wave in the solid portion was estimated as twice that of the longitudinal wave, considering the properties of polymeric materials [31]. The parameters used in this simulation are shown in Table 1. The FDTD simulation software was originally programmed by our group [32].

\subsection{Numerical Results}

Figures 7 show the screenshots of the distribution of sound pressure (in liquid portion) or root-mean-square value of normal stresses (in solid portion) at the central $x-y$ plane of the three-dimensional simulation field. Figures (a) to (c) show the results without specimen, (d) to (f) show the results with specimen. The separation of fast wave and slow wave can be seen [18].

The calculated waveforms are shown in Figs. 8(a) and (b). Figure 8(a) shows the result when the transmitted wave passes only through the water. The slight vibration at the tail of the waveform in Fig. 8(a) seems to result from the spatial resolution in simulating. Figure 8(b) shows the result when the wave is focused on the center portion of the specimen, which is in a simular condition to that of Fig. 3(b). The estimated BV/TV (bone volume/total tissue volume, or bone volume fraction) around the area of this result was $32.9 \%$. Here, the BV/TV is the averaged value of the cuboidal area in the model of $3 \times 3 \times 9 \mathrm{~mm}^{3}$ around the focal point of the concave source. This cuboidal ROI (region of interest) was estimated considering the focal area of transmitted wave in experiments [7]. Here, both fast and slow waves can be seen. The fast wave is considered to propagate mainly in the solid portion of bone specimen. The amplitude of the fast wave was smaller than that of the slow wave, which has also been reported from experimental studies [2-4].

\section{DISCUSSION}

In these simulations and experiments, we obtained the peak amplitudes of the fast and slow waves and the speed of 

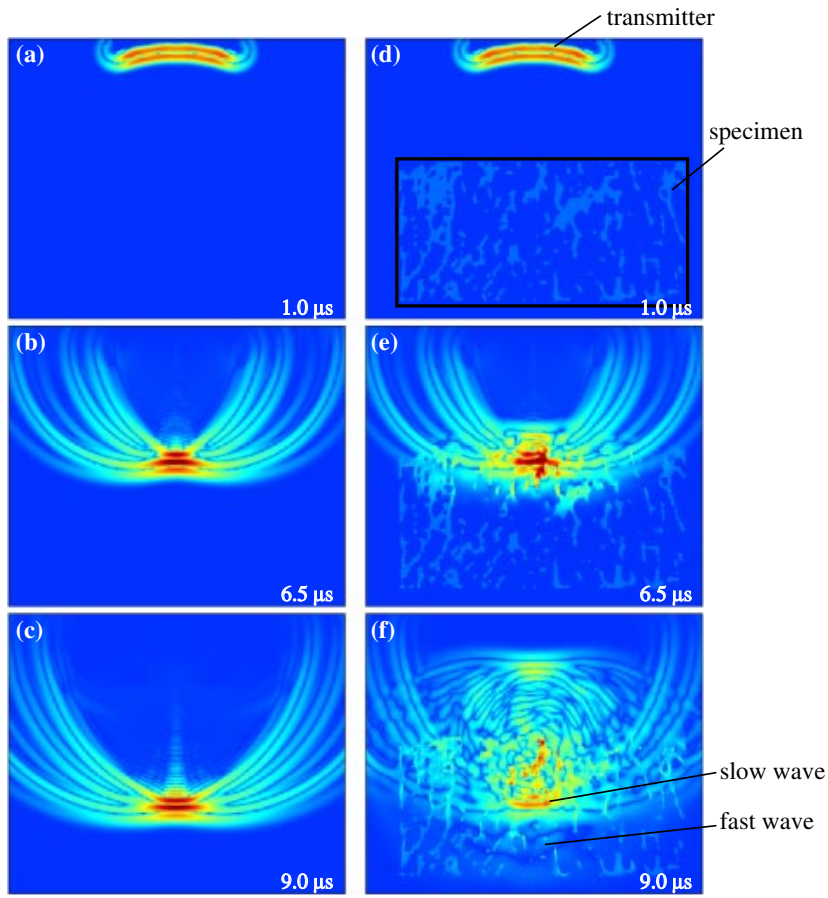

Fig. 7 Screenshots of the distribution of sound pressure in the $x-z$ plane of the three-dimensional simulation field inluding the specimen center. Figures (a) to (c) show the results without specimen, (d) to (f) show the results with specimen.
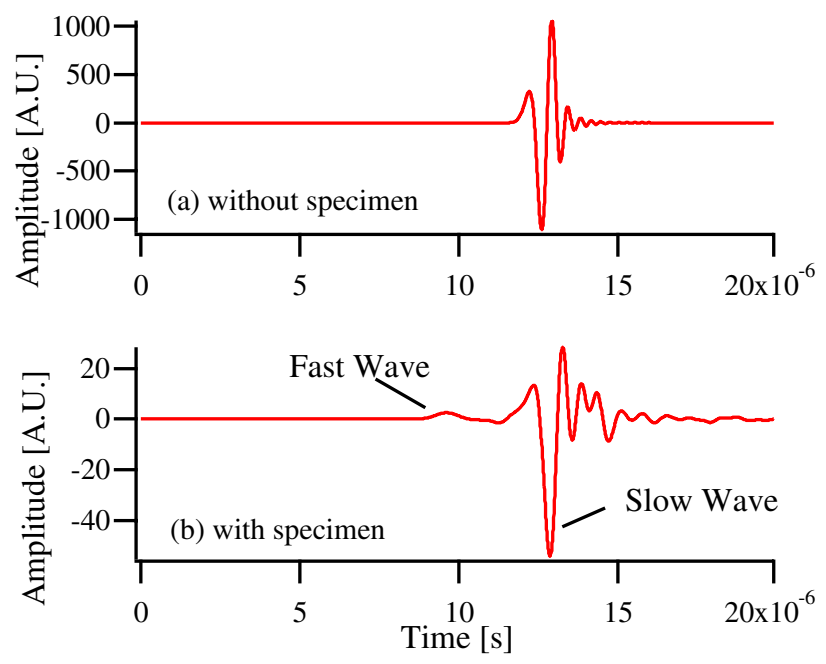

Fig. 8 Simulated waveforms. Waves pass through the simulation model, (a) without the specimen, or (b) with the specimen.

the fast wave estimated from the arrival time of the wave front. Here, we adopted the first positive peak as the fast wave amplitude and the maximum amplitude of whole waveform as the slow wave amplitude. In this study, we could not obtain the speed of slow waves because superposition with fast waves affected the wave fronts of the slow waves.

Figure 9(a) shows the relationship between BV/TV around the focal point and the speed of the fast wave. We can see correlations in both the simulated and experimental results. Both results increased in proportion to the BV/TV. This indicates that the fast wave speed depends on the amount of trabecula in the wave propagation path.

The relationships between the $\mathrm{BV} / \mathrm{TV}$ and the peak amplitude of the fast and slow waves are shown in Figs. 9(b)(c). Here, all values are normalized by the peak amplitude of the wave that passed only through the water. The amplitude of the fast wave has a positive correlation to the $\mathrm{BV} / \mathrm{TV}$, while the amplitude of the slow wave has a negative correlation. The fast wave passes mainly through the solid portion (trabeculae) and the slow wave passes mainly through the liquid portion [2-4]. The increase in the $\mathrm{BV} / \mathrm{TV}$ results in a minimization of liquid path or the generation of closed liquid portions surrounded by solid bone, which rapidly increases the amplitude of the fast wave and contrarily decreases that of the slow wave. These characteristics are also supported by the former experimental studies $[2,3,7]$.

Table 2 shows the correlation coefficients of the BV/ $\mathrm{TV}$ and each indicator of the simulated and experimental results. These data show clear correlation between simulations and experiments: The correlation coefficients of the fast wave speed, the amplitudes of the fast and slow waves between simulation and experiments were $0.58,0.74$, and $0.83(p<0.01)$, although we adopted the velocity and attenuation values of cortical bone, because the values in the trabeculae are unknown. There seem to be other causes for these differences in addition to the velocity and attenuation values. For example, the selection of the threshold for the modeling of CT image [16,33] and present assumption of isotropic elasticity in the trabeculae. We also should point the difference of simulation field from the measurement due to memory problem: The transmitter in the simulation was smaller than that in the actual experimental system, which results in wider beam width at the focal area because the ratios of the wavelength and the radius of the transducer are disparate. In addition, due to the limitation of simulation field, we put the smaller receiver near the specimen. We can also point out the effect of the frequency dispersion of attenuation coefficients in trabeculae, and the influence of boundary friction at the interface of the liquid and solid portions [17]. These problems should be considered in future studies.

Despite the problems, however, the simulation reveals some interesting results. Figure 9(d) shows the relationships between the $\mathrm{BV} / \mathrm{TV}$ and the peak amplitude ratio between fast and slow waves. Differences at low BV/TV area in Fig. 9(c) and high BV/TV area in Fig. 9(b) disappeared. Some agreements between the simulated and experimental results becomes apparent. The amplitude ratio concerns the separation mechanism of the initial wave into the fast and slow waves; therefore, this agreement of simulation and experiment suggests the possibility of 

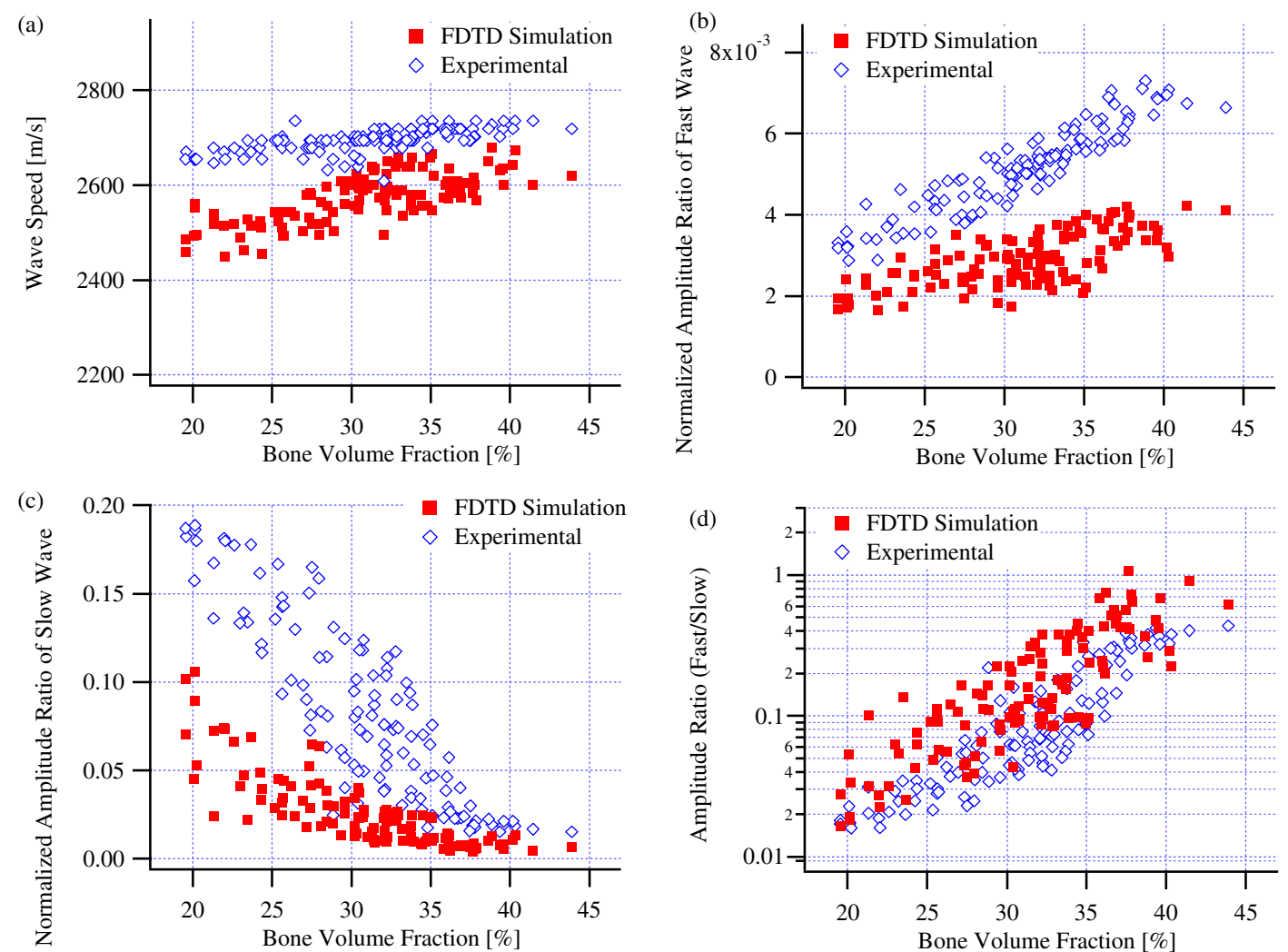

Fig. 9 Relationship between BV/TV (bone volume fraction) and (a) speed of fast wave, (b) peak amplitude of fast wave, (c) peak amplitude of slow wave, and (d) peak amplitude ratio of fast and slow waves. The longitudinal wave speed was assumed to be $4,400 \mathrm{~m} / \mathrm{s}$. All values are normalized by the peak amplitude of the wave that passed through the water only. The same unique specimen was used for both the simulation and the experiment.

Table 2 Correlation coefficients $(R)$ of (i) BV/TV v.s. simulated results, (ii) BV/TV v.s. experimental results, and (iii) simulated results v.s. experimental results. ( $p<0.01$ in all cases).

\begin{tabular}{|c|c|c|c|}
\hline Indicator & $\begin{array}{c}\text { (i) } \mathrm{BV} / \mathrm{TV} \\
\text { v.s. Simulation }\end{array}$ & $\begin{array}{c}\text { (ii) } \mathrm{BV} / \mathrm{TV} \\
\text { v.s. Experiment }\end{array}$ & $\begin{array}{l}\text { (iii) Simulation } \\
\text { v.s. Experiment }\end{array}$ \\
\hline Wave speed & 0.71 & 0.66 & 0.58 \\
\hline Normalized amplitude ratio of fast wave & 0.69 & 0.93 & 0.74 \\
\hline Normalized amplitude ratio of slow wave & -0.81 & -0.87 & 0.83 \\
\hline
\end{tabular}

understanding the generation mechanism of wave separation by FDTD simulation using X-ray CT images. The separation phenomena of longitudinal wave into fast and slow waves seems to depends on the $\mathrm{BV} / \mathrm{TV}$.

Figures 10 show the results when the londitudinal wave speed were assumed to be $4,060 \mathrm{~m} / \mathrm{s}$, where the Poisson's ratio was 0.34. A comparison of Figs. 9 and 10 clarifies that the selection of the longitudinal wave speed in the trabeculae affects the results: a higher longitudinal wave speed results in not only the higher fast wave speed but also the smaller fast wave amplitude and larger slow wave amplitude. These results are believed to be caused by the change in the acoustic impedance of trabeculae, suggesting the importance of the initial properties used in the simulation. The comparison of Figs. 9 and 10 tell us that more special attenuation to the wave properties for simulation will give us better correlation between simulation and experiments.

\section{CONCLUSION}

Using a 3-D X-ray CT model of a bovine cancellous bone, the generation of fast and slow waves was confirmed by the elastic FDTD simulation. We also experimentally measured the waves propagated in an identical bone specimen under the same conditions. Comparing the results in detail, we found the similar tendency between the simulated and experimental results.

The simulated speed of fast wave, the peak amplitude of fast and slow waves, and the amplitude ratio between those two waves showed clear correlations with BV/TV. The discrepancy of values between experiments and simulation seems to come from the modeling difficulties 

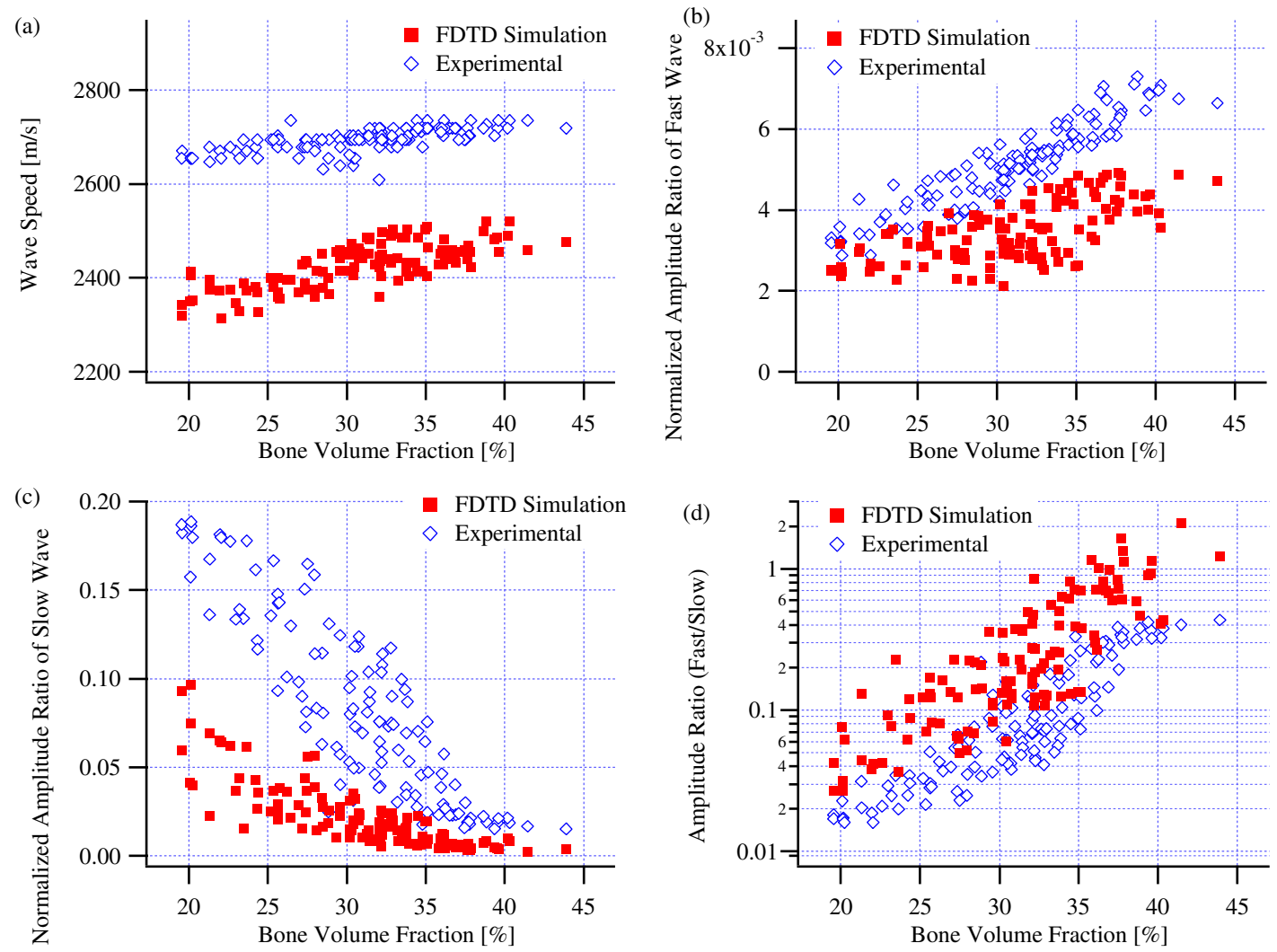

Fig. 10 Relationship between BV/TV (bone volume fraction) and (a) speed of fast wave, (b) peak amplitude of fast wave, (c) peak amplitude of slow wave, and (d) peak amplitude ratio of fast and slow waves. The longitudinal wave speed was assumed to be $4,060 \mathrm{~m} / \mathrm{s}$. The Poisson's ratio was 0.34 , which is same as the condition of Fig. 9 . All values are normalized by the peak amplitude of the wave that passed through the water only. The same unique specimen was used for both the simulation and the experiment.

(adopted values for wave properties, friction loss, etc.). However, the peak amplitude ratio between the fast and slow waves agreed with the experimental results. This simulation technique can help us understand wave propagation in a complicated medium and can be an effective tool for designing a new in vivo ultrasonic measurement system for osteoporosis.

\section{ACKNOWLEDGMENTS}

The authors like to thank Dr. Masahiko Takada at Shiga University of Medical Science for the X-ray microfocus CT.

This study was partly supported by a bilateral joint project between the Japan Society for the Promotion of Science (JSPS) and Centre National de la Recherche Scientifique (CNRS) as well as the Academic Research Frontier project of Doshisha University and the Ministry of Education, Culture, Sports, Science and Technology of Japan. In addition to the Grant-in-Aid for Young Scientists (B) (20791218) supported by JSPS, some parts of this study were also supported by the Open Competition for the Development of Innovative Technology, a project of the Ministry of Education, Culture, Sports, Science and Technology.

\section{REFERENCES}

[1] C. F. Njeh, D. Hans and T. Fuerst, Quantitative Ultrasound: Assessment of Osteoporosis and Bone Status, 1st ed. (Taylor \& Francis, London, 1999).

[2] A. Hosokawa and T. Otani, "Ultrasonic wave propagation in bovine cancellous bone," J. Acoust. Soc. Am., 101, 558-562 (1997).

[3] A. Hosokawa, T. Otani, T. Suzaki, Y. Kubo and S. Takai, "Influences of trabecular structure on ultrasonic wave propagation in bovine cancellous bone," Jpn. J. Appl. Phys., 36, 3233-3237 (1997).

[4] T. Otani, "Quantitative estimation of bone density and bone quality using acoustic parameters of cancellous bone for fast and slow waves," Jpn. J. Appl. Phys., 44(6B), 4578-4582 (2005).

[5] I. Mano, K. Horii, S. Takai, T. Suzaki, H. Nagaoka and T. Otani, "Development of novel ultrasonic bone densitometry using acoustic parameters of cancellous bone for fast and slow waves," Jpn. J. Appl. Phys., 45(5B), 4700-4702 (2006).

[6] I. Mano, T. Yamamoto, H. Hagino, R. Teshima, M. Takada, T. Tsujimoto and T. Otani, "Ultrasonic transmission characteristics of in vitro human cancellous bone," Jpn. J. Appl. Phys., 46(7B), 4858-4861 (2007).

[7] K. Mizuno, M. Matsukawa, T. Otani, M. Takada, I. Mano and T. Tsujimoto, "Effects of structural anisotropy of cancellous bone on speed of ultrasonic fast waves in the bovine femur," IEEE Trans. Ultrason. Ferroelectr. Freq. Control, 55, 14801487 (2008). 
[8] M. A. Biot, "Generalized theory of acoustic wave propagation in porous dissipative media," J. Acoust. Soc. Am., 34, 12541264 (1962).

[9] K. A. Wear, "A stratified model to predict dispersion in trabecular bone," IEEE Trans. Ultrason. Ferroelectr. Freq. Control, 48, 1079-1083 (2001).

[10] A. Hosokawa, "Simulation of ultrasound propagation through bovine cancellous bone using elastic and Biot's finite-difference time-domain methods," J. Acoust. Soc. Am., 118, 1782 1789 (2005).

[11] T. J. Haire and C. M. Langton, "Biot theory: A review of its application to ultrasound propagation through cancellous bone," Bone, 24, 291-295 (1999).

[12] S. Chaffai, F. Padilla, G. Berger and P. Laugier, "In vitro measurement of the frequency-dependent attenuation in cancellous bone between 0.2 and $2 \mathrm{MHz}$," J. Acoust. Soc. Am., 108, 1281-1289 (2000).

[13] J. J. Kaufman, G. Luo and R. S. Siffert, "Ultrasound simulation in bone," IEEE Trans. Ultrason. Ferroelectr. Freq. Control, 55, 1205-1218 (2008).

[14] E. Bossy, F. Padilla, F. Peyrin and P. Laugier, "Threedimensional simulation of ultrasound propagation through trabecular bone structures measured by synchrotron microtomography," Phys. Med. Biol., 50, 5545-5556 (2005).

[15] F. Padilla, E. Bossy, G. Haïat, F. Jenson and P. Laugier, "Numerical simulation of ultrasound transmission in cancellous bone," 2005 IEEE Ultrason. Symp., Vol. 4, pp. 2022-2025 (2005).

[16] G. Haïat, F. Padilla, F. Peyrin and P. Laugier, "Variation of ultrasonic parameters with microstructure and material properties of trabecular bone: A 3D model simulation," J. Bone Miner. Res., 22, 665-674 (2007).

[17] Y. Nagatani, H. Imaizumi, T. Fukuda, M. Matsukawa, Y. Watanabe and T. Otani, "Applicability of finite-difference timedomain method to simulation of wave propagation in cancellous bone," Jpn. J. Appl. Phys., 45(9A), 7186-7190 (2006).

[18] Y. Nagatani, K. Mizuno, T. Saeki, M. Matsukawa, T. Sakaguchi and H. Hosoi, "Numerical and experimental study on the wave attenuation in bone - FDTD simulation of ultrasound propagation in cancellous bone -," Ultrasonics, 48, 607-612 (2008).

[19] F. Padilla, E. Bossy and P. Laugier, "Simulation of ultrasound propagation through three-dimensional trabecular bone structures: Comparison with experimental data," Jpn. J. Appl. Phys., 45(8A), 6496-6500 (2006).

[20] F. Jenson, F. Padilla, V. Bousson, C. Bergot, J.-D. Laredo and P. Laugier, "In vitro ultrasonic characterization of human cancellous femoral bone using transmission and backscatter measurements: Relationships to bone mineral density," $J$. Acoust. Soc. Am., 119, 654-663 (2006).

[21] T. Otani, "Quantitative estimation of bone density and bone quality using acoustic parameters of cancellous bone for fast and slow waves," Jpn. J. Appl. Phys., 44(6B), 4578-4582 (2005).

[22] K. S. Yee, "Numerical solution of initial boundary value problems involving Maxwell's equations in isotropic media," IEEE Trans. Antenna Propag., AP-14, 302-307 (1966).

[23] E. F. Toro, J. F. Clarke and E. F. Toro, Numerical Methods for Wave Propagation (Kluwer Academic Pub., Norwell, Mass., 1998).

[24] R. L. Higdon, "Absorbing boundary conditions for difference approximations to the multi-dimensional wave equation," Math. Comput., 47, 437-459 (1986).

[25] M. Sasso, G. Haïat, Y. Yamato, S. Naili and M. Matsukawa, "Frequency dependence of ultrasonic attenuation in bovine cortical bone: An in vitro study," Ultrasound Med. Biol., 33,
1933-1942 (2007).

[26] M. Sasso, G. Haïat, Y. Yamato, S. Naili and M. Matsukawa, "Dependence of ultrasonic attenuation on bone mass and microstructure in bovine cortical bone," J. Biomech., 41, 347355 (2008)

[27] Y. Yamato, H. Kataoka, M. Matsukawa, K. Yamazaki, T. Otani and A. Nagano, "Distribution of longitudinal wave velocities in bovine cortical bone in vitro," Jpn. J. Appl. Phys., 44(6B), 4622-4624 (2005).

[28] Y. Yamato, M. Matsukawa, T. Otani, K. Yamazaki and A. Nagano, "Destribution of longitudinal wave properties in bovine cortical bone in vitro," Ultrasonics, 44, e233-e237 (2006).

[29] Y. Yamato, M. Matsukawa, T. Yanagitani, K. Yamazaki, H. Mizukawa and A. Nagano, "Correlation between hydroxyapatite crystallite orientation and ultrasonic wave velocities in bovine cortical bone," Calcif. Tissue Int., 82, 162-169 (2008).

[30] M. Sakamoto, M. Kawabe, M. Matsukawa, N. Koizumi and N. Ohtori, "Measurement of wave velocity in bovine bone tissue by micro-Brillouin scattering," Jpn. J. Appl. Phys., 47, 42054208 (2008).

[31] A. W. Nolle and P. W. Sieck, "Longitudinal and transverse ultrasonic waves in a synthetic rubber," J. Appl. Phys., 23, 888-894 (1952).

[32] Y. Tanikaga, T. Sakaguchi and Y. Watanabe, "A study on analysis of intracranial acoustic wave propagation by the finite difference time domain method," Proc. Forum Acusticum, Sevilla (2002)

[33] Y. Nagatani, H. Imaizumi, T. Fukuda, M. Matsukawa, Y. Watanabe and T. Otani, "FDTD simulation on the wave propagation in the cancellous bone," 8 e Congres Francaise d'Acoustique - Proc., pp. 681-684 (2006).

Yoshiki Nagatani received B.E., M.E., and Ph.D. degrees in Engineering from Doshisha University in 2001, 2003, and 2006, respectively. He became a research fellow at the Department of Otorhinolaryngology-Head and Neck Surgery, Nara Medical University in 2006. He is currently a lecturer of the Department of Electric Engineering in Kobe City College of Technology.

Katsunori Mizuno received B.E. and M.E. degrees in Engineering from Doshisha University in 2004 and 2006, respectively. He currently works for Panasonic corporation.

Takashi Saeki received B.E. in Engineering from Doshisha University in 2007. He is a candidate of M.E. degree in Engineering at Doshisha University. He founded a venture company of information network technology and currently works for the company as a CEO.

Mami Matsukawa joined AIST (National Institute of Advanced Industrial Science and Technology) MITI (Ministry of International Trade and Industry). She received the Dr.Eng. degree from Doshisha University in 1993. Currently, she is a professor at the Faculty of Life and Medical Sciences, Graduate School of Engineering, Doshisha University.

Takefumi Sakaguchi received B.E., M.E., and Ph.D. degrees in Engineering from Doshisha University in 1997, 1999, and 2002, respectively. He is an Assistant Professor at the Department of Otorhinolaryngology-Head and Neck Surgery, Nara Medical University since 2002 .

Hiroshi Hosoi graduated from Nara Medical University in 1975 and received Dr. of Medical Science degree from Kinki University in 1985. He worked for Kinki University as a research associate and associate professor. He is currently a chairman and professor in the Department of Otorhinolaryngology-Head and Neck Surgery, Nara Medical University since 1999. 